\title{
P02-204
}

\section{FIBROMYALGIA: RELATIONSHIP BETWEEN PAIN AND MOOD}

\author{
A. Barreiro ${ }^{1}$, A. Arroita ${ }^{1}$, K. Ugarte ${ }^{1}$, A. Arnaiz ${ }^{1}$, A. Sarasola ${ }^{1}$, E. Zallo ${ }^{1}$, J. Perez Agote ${ }^{1}$, A.A. Gandarias ${ }^{2}$ \\ ${ }^{1}$ Psychiatry, Hospital of Zamudio, ${ }^{2}$ Hospital Psiquiátrico De Zamudio, Zamudio, Spain
}

Introduction: Fibromyalgia is an entity with idiopathic etiology affecting $2-4 \%$ of population. It is a frequent cause of disability, its most usual symptoms being diffuse pain and fatigue. There is evidence that alterations in several chemical and hormonal factors could explain changes in pain perception mechanisms, accounting for some of the symptoms. However, the high incidence of comorbid psychiatric disorders tends to indicate there is an associated psychopathology in fibromyalgia patients, especially regarding affectivity. Some authors believe fibromyalgia could be considered an evolution of the concept of neurasthenia already defined by Beard in 1860.

Materials and methods: Comprehensive review of the scientific literature (Medline, Psychoinfo, Medex) on fibromyalgia and pain mechanisms published over the last three years.

Results: Mood regulation has been linked to pain perception through neurotransmitters and brain structures. Also, several studies state that depression changes the neuroendocrine, immune and processing regulation of pain.

However, some researchers believe there is enough evidence for a psychiatric approach to fibromyalgia, as there is a relationship between depression and fibromyalgia. Thus, fibromyalgia could be considered an affective disorder.

Conclusion: Current research does not clearly show whether pain precedes affective alteration or the other way round, or whether mood and pain depend on common mechanisms. 